\title{
SISTEMA EXCRETOR DO FÍGADO DE VEADOS \\ (Manzana americana, Blastocerus bezoarticus e Manzana simplicicornis) II. SISTEMATIZAÇÃO DO ramus principalis sinister
}

\author{
EXCRETORY SYSTEM OF THE LIVER IN DEERS (Manzana americana, Blastocerus bezoarticus e Manzana \\ simplicicornis) \\ 11. Ramus principalis sinister
}

Maria Angélica MIGLINO'; Wilson Machado SOUZA²; Liberato J. Afonso DIDIO’.

\begin{abstract}
RESUMO
A sistematização do ramus principalis sinister do sistema excretor do fígado foi estudada em 8 peças retiradas de 6 veados mateiros (Manzana americana) I veado catingueiro (Manzana simplicicornis) e 1 veado do rabo branco (Blastocerus bezoarticus). Foi utilizado látex Neoprene corado para o preenchimento das vias biliares, e o método empregado pára a evidenciação dos colctores foi o da dissecação, realizada após a fixação das peças com solução aquosa de formol a 10\%. Nas três espécies, participam do sistema do ramus principalis sinister sob diferentes arranjos, o ramus dorsalis lobi sinistrim o truncus intermediomedialis, o truncus intermediolateralis, o ramus lateralis lobi sinistri e o ramus lobi quadrati. Do sistema de ductos ainda fazem parte contribuições inominadas, vindas dos vários territórios hepáticos.
\end{abstract}

UNITERMOS: Fígado: Veados; Vias biliares.

\section{INTRODUÇÃO}

Este trabalho tem por meta a obtenção do conhecimento dos padrões de distribuição do ramus principalis sinister do sistema excretor do fígado dos veados (Manzana americana, Manzana simplicicornis Blastocerus bezoarticus).

\section{MATERIAL E MÉTODO}

Para a realização deste trabalho utilizamos 8 fígados retirados de 6 veados mateiros (Manzana americana), 3 machos e 3 fêmeas, de 1 veado catingueiro (Manzana simplicicornis) macho e de l veado do rabo branco (Blastocerus bezoarticus) macho, sendo todos adultos e provenientes da região do Pantanal Matogrossense.

Em cada peça (fígado e trato duodenal correspondente à desembocadura do colédoco) as vias bilíferas foram injetadas com látex Neoprene corado. A seguir, procedeu-se à fixação do material em solução aquosa de formol a $10 \%$ durante 48 horas, à dissecação e à documentação.

Adotamos no relato dos resultados, o mesmo critério de designação utilizado por JABLAN-PANTIC 5 (1963).

\section{RESULTADOS}

\section{Veado Mateiro (Manzana americana).}

As 6 peças de veados mateiros, após injeção e dissecação demonstram que o ductus choledocus, constantemente isento de tributários, resulta da reunião do ramus principalis dexter e do ramus principalis sinister.

O ramus principalis sinister origina-se da convergência do ramus dorsalis lobi sinistri, truncus intermedio medialis, truncus intermedio lateralis, ramus lateralis lobi sinistri e ramus lobi quadrati, os quais, ao constituí-lo, assume diferentes arranjos.

O ramus medialis lobi sinistri une-se ao truncus intermediomedialis para compor via comum em 3 dos fígados estudados (Obs. If, 3f, 6m), quando ambos encontram-se desprovidos de eferentes, l vez (Obs. If) e, apenas ao primeiro deles afluindo tributário isolado, do lobus sinister (território medial), l vez (Obs. 3f) e do lobus caudatus (pars supraportalis), 1 vez (Obs. $6 \mathrm{~m}$ ). O tronco comum assim formado encontra-se livre de afluentes 1 vez (Obs. 3f) e recebe-os nos outros 2 casos, provindos do lobus sinister (território intermédio), 1 vez (Obs. If), e do lobus caudatus (pars supraportalis), 1 vez (Obs. 6m).

Professor Associado - Faculdade de Medicina Veterinária e Zootecria da USP

- Professor Adjunto - Faculdade de Medicina Veterinária, Universidade Estadual Paulista - Campus de Araçatuba - SP

- Professor Visitante - Escola Paulistu de Medicina - São Palulo - SP. e Professor Emérito do Medical College of Ohio. USA 


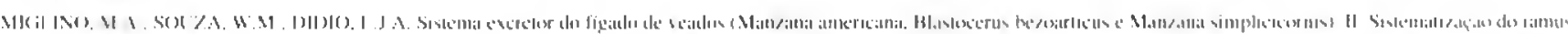

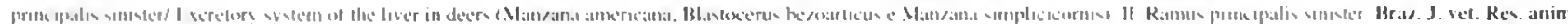

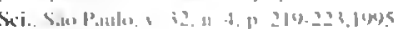

() truncus intermediomedialis une-se an truncus intermediolateralis. formando tronco comum em 3 órgäos (c)h. 2m. 4m. 51). nos quais. ambos recebem tributarios apenals I ver (obs. 2m) e vindos do lobus sinister. vale diser, alo primeroro componente chega isolado do cerritorio dorsal e an segundo. do território lateral: apenas o segundo deles recebe alerentes l ve robs. fill). ou seja. um elemento isolado do lobus sinister (territorio intermédio): somente an primeiro vat ler alerente. I ves (0)bs. 50). isto é. tribulário inclependente do lobus sinister (territorio intermédio). A via commun. constituida pela alluência do truncus intermediomedialis mais truncus intermediolateralis. É vista live de elerenter 2 reas (obs. tin. 50 ) e recebendo elemento isolado do lobus simister (território lateral). I vez. (Obs. $2 \mathrm{~m})$.

() ramus lateralis lobi sinistri conllui para o tronco comum. composto pelo truncus intermediomedialis : truncus intermediolateralis em 2 dos 6 órgãos estudados (O)b. 2m. 51). quando é visto permanentemente isento de afluentes, assim como a via por eles formada. O ramus lateralis tobi sinistri comverge com o truncus intermediolateralis. para formarem ducto comum em 2 glândulas (Ohs. If. om), oportunidade em que ambos estão lives do coletores. I vey (obs. (om). a collhendo-os. entretanto. I vey. (O). 11), sendo cles oriundos do lobus sinister. isto é. do erritorio dorsal. para o primeiro deles e do intermedio, para o segundo. A via comum observada neste arranjo surge desprovida de tributários. I ves (Ohs. (6m) e no caso complementar recebe-0) $1 \mathrm{k} /$ lobs. 11). do lobus sinister (terrilório lateral). () aludido ramus lateralis lobi dextri, unc-se a) ramus lobi quadrati. I ve/ (Obs. 30). quando ambos recebem confluentes. isto s. ao primeiro deles. chegam dois clementos (b) lobus sinister. un do territorio intermédio o outro do dorsal e an segundo, outro ducto dessa mesma origem. do território latcral. () ducto comum aqui observado achat-se isento de atluenles. Também o ramus lateralis lobi dextri comverge para via comum composta pelo ramus dorsalis lobi sinistri. truncus intermedialis mais truncus intermediolateralis. en 1 lighado (obs. $4 \mathrm{~m}$ ). oportunidade em que recebe um dos rami intermedii lobi sinistri. () ducto resultante desta união recebe tronco comum de dois componentes, um do lobus sinister (território latteral), outro do lobus quadratus a afluente isolado do lobus caudatus (pars supraportalis).

() ramus medialis lobi sinistri une-se alo ducto composto pelo truncus intermediomedialis. truncus intermediolateralis c ramus lateralis lobi sinistri. cm 2 órgàos (Obs. $2 \mathrm{~m}$. 51). yuando surge live de eferentes em ambas as peças. A via resultante dessa ansocialçan recebe tronco common an coletor procedente do lobus simister (território lateral) e do lobus enadratus alén de alluente isolado do lobus couda- tus (pars supraportalis). I ves. (()bs. $2 \mathrm{~m}$ ) e appenals um componente isolado desta tilima procedêncial. I ves (ohs. 51 ).

() ramus quadrati une-se ao tronco lomado pela contluencia do ramus medialis lobi sinistri. truncus intermediomedialis mais truncus intermediolateralis a ramas lateralis

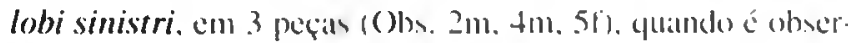

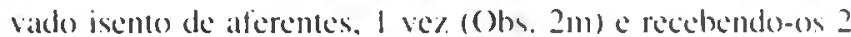
veres (obs. tm. 50), ou sejal, um colatcral isolado vindo do lobus sinister (temitónio laterall) no primeiro calso co outro do lobus dexter (território intcrmédion), mo segundo caso. A s ia composta nestes casos. já representar o propron ramas principalis sinister a surge live de contribuiçós. I ve\% (O)h 50) e acollendo, tributário isolado do tobus caudatus (pars supraportalis) has outras 2 preparaçōes. () ramus lobi quadrati. vaj ter a via comum. resultante da confluência do ramus medialis lobi dextri mais truncus intermediomedialis. Iruncus intermediolateralis mais ramus lateralis lobi sinistri. cm 2 preparaçoes (O)b. If, 6mo). oportunidade em que surge live de aferentes. O dacto fomado após a conlluência do aludido ramo ó o ramus principalis sinister quo recebe tronco comum a quatro sias inominadas. vale dizer. dois tributários do lobus caudatus (pars supraportalis). um do lobus sinister (cerritório medial) é outro do lobus quadratus. I vez (Obs. li) e acolhe. Na outra glândulat, dois alluentes do lobus caudatus (pars supraportalis). cm tronco comum (obs. (om).

() truncus intermediolateralis contlui para o aronco resultante da união do ramus medialis lobi sinistri c truncus intermediomedialis am 1 fígado (o). 1). quando é visto isento de aferentes. () decto assim formado recebe dois alluentes do lobus caudatus (pars supraportalis) e. a seguir. comverge com a via comum formada pela uniāo do ramus lateralis lobi sinistri com o ramus lobi quadrati. para compor o ramus principalis sinister. isento de afluen-

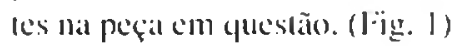

\section{Veado do Rabo Branco (Blastocerts bezoarticus)}

Neste caso, o ramus medialis lobi simistri unc-se an truncius intermediomedialis para compor via comum, yuando alo último destes vêm ter dois alerentes isolados do lobus simister, sendo o primeito. um dos rami intermedii lobi sinistri c. o segundo. procedente do território dorsial. () tronco comum acui observado, encontra-se live de tributários. () truncus intermediolateralis une-se a via comum an ramus medialis lobi sinistri c alo truncus intermediomedialis compondo tronco comum; nesta preparação. lanto o truncus intermediolateralis como o tronco formade após sua convergênciatencontram-se desprovidos de alluentes. () ramus lateralis lobi simistri altere a via já descrila. yuando acolhe afluen Ic isolado do lobus quadratus a dois dos rami intermedii 


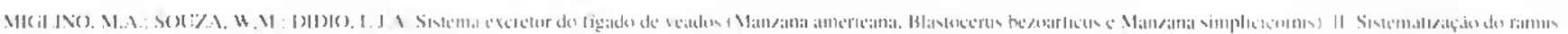

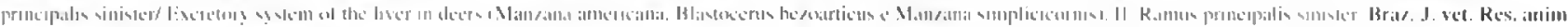

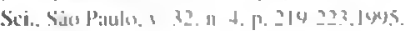

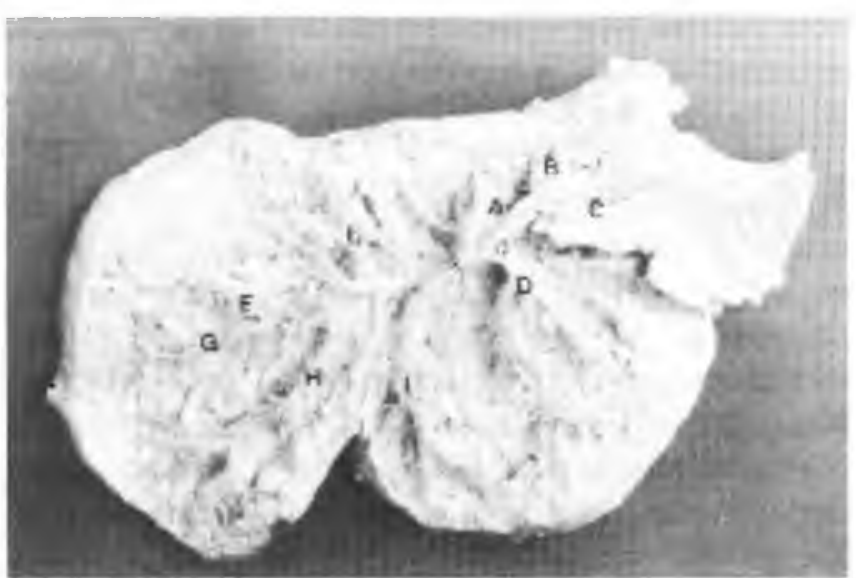

FIC; R:I I

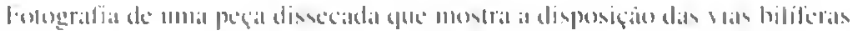

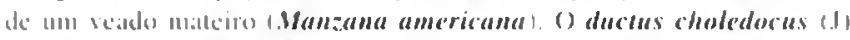

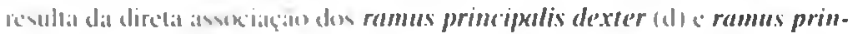

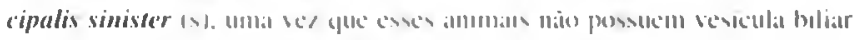

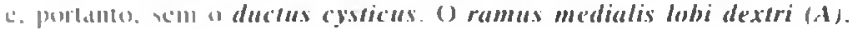
ramus dorsalis lobi dextri (B): o ramus processi coudani (C) I c a ramus

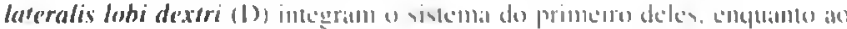

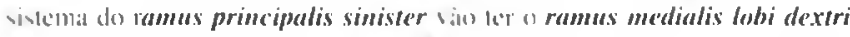
11:1. o truncus intermedionedialis (1-1. truncus intermedioluteralis (Ci., ramus lateralis lobi simistri (H) c oramus lobi quadrati Il].

lobi sinistri em tronco comum: o decto formado após a sla alfuencial acolle dois eferentes oritundos do lobus caudatus (pars supraportalis). () ramms lobi quadrati converage para () ducto comum formado pelo ramus medialis lobi sinistri mais truncus intermediomedialis. truncus intermediolateralis c ramus lateralis lobi sinistri, o ducto atssim constituído acolhe altuente inolado do lobus caudatus (pars supraportalis) c comstitui já a propprio ramus principalis sinister (Fig. 2)

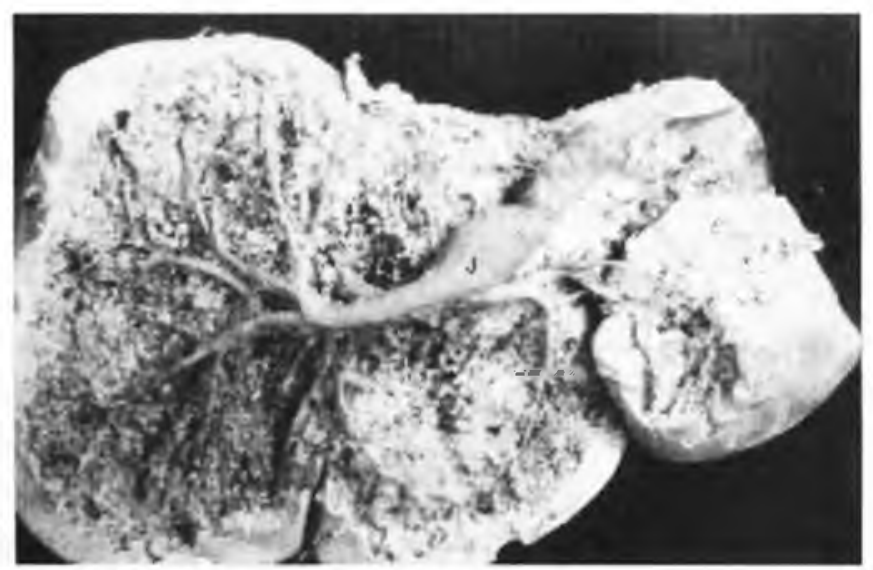

]:IC, I IR: 2

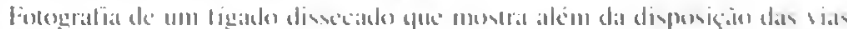

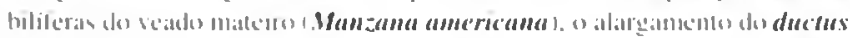

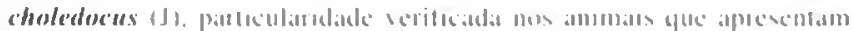

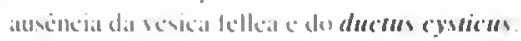

\section{Veado Catingueiro (.Manzana simplicicornis)}

Xisce ligakdo, o truncus intermediomedialis unc--xe an truncus intermediolateralis, yuando apenass o segunde delen secebo componkente inominado do lohus simister (kerritorio dorsall): 6) dec'lo comum formado pela reunião dos compenconces mominados a viso listo de allewentes. () ramms lateralis lobi simistri conserge paral a via anteriormente descritat, opxontunidade em que recebe dois dos rami intermedii lobi sinistri. entre on yualis se poxicional outro componente isolindo do lobus quadratus: a via comum resultante da convergéncial destes componten-

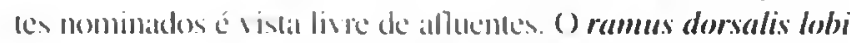
sinistri allui paral o duclo comprosto pelo truncus intermediomedialis mais. truncus intermediolateralis c ramus lateralis lobi sinistri. acollhendo dois atluentes inominados independen-

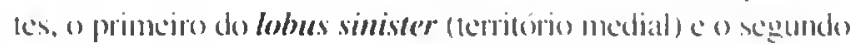
do lobus caudatus (pars supruportalis).

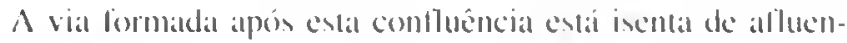
les. () ramus lobi quadrati converge paltat o duclo anteriormente descrito, encontrande-se live de yualyuer tributário: já a via observada apón estal alluéncia. represental o proprio ramus principalis sinister. o qual acolhe trihutáro isolado do lobus caudatus tpars supraporalis), seguido de dois altuentes. em troncos, oriundos do lobus quadratus. (Fig. 3).

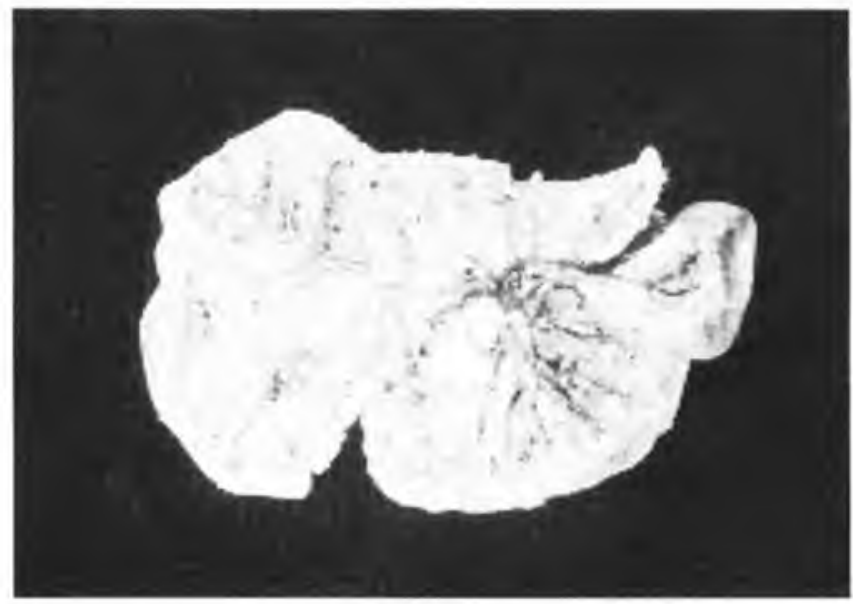

FIs:tR. 3

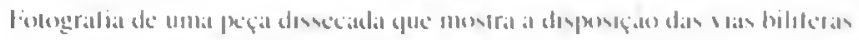

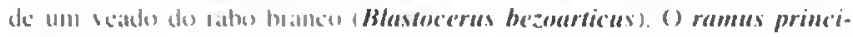

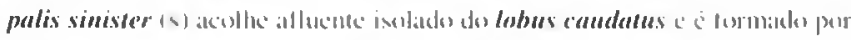

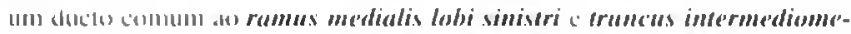
dialis, mencus imermediolateralis to rames lateralis lobi smiveri. " yual recelbe brames lobi quadrati

\section{I)ISCUSSÃO}

A composiçăo do romms principalis simister. de acordo com!

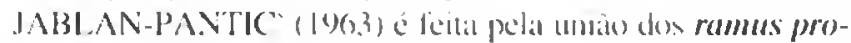
cessi papillaris. ramus lobi quadrati. ramus lateralis bohi sinisuri. ramus medialis tobi siniseri c rumi medii lobi sinistri. 


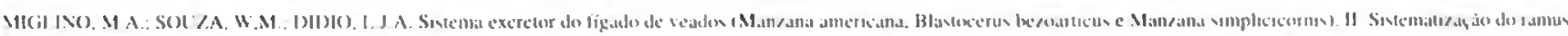

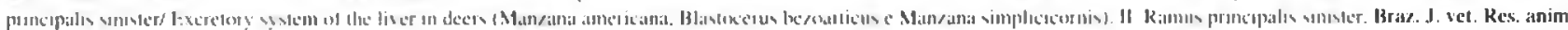

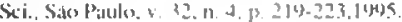

Componentes inominados não foram registrados por JABI.AN-PAN'TIC" (1963) . mas foram verificados por PAIVA: D'ERRICO'(1959), ANNUN\%IATA' (1969). PRAI)A* (1971). D'ERRICO'(1976). PRAI)A c al." (1976, 1985), SOU\%,A Et al." (1985), SOLLA"'(1984. 1989). BASTOS NETO( ): PRAD) (1989) os (quais pesquisaram o mesmo assunto no lígado de ruminantes, como participantes diretos ou indiretos do ramus principalis sinister. fato ora patenteado nos tigados dos veados.

(iE:TTY' (1975), a0 aludir a pecpuenos ductos bilileros que se reúnem de diferentes maneiras. para compor os ductos bilíferos dos lobos hepáticos direito e espuerdo. provavelmente estará se referindo a estes componentes que acabamos de relatar. OTTAVIANI" (1933) indica que os ductos biliferos resultam da fusão de variável número de afluentes primários. secundários e terciários, mas sem fornecer meios para uma conceituação destes afluentes. reduz as possibilidades de uma comparação mais estreita com os dados agora observiados. Os alluentes terciários estabelecidos pelo autor. provavelmente correspondem aos componentes inominados vindos do lobus caudatus, lobus sinister e lobus quadratus, ora também evidenciados nos cervos.

Como é fácil verilicár, nas peças estudadas, devido à ausência da vesicula biliar e do ductus cysticus reduz-se a apenas uma a possibilidade de origem do ductus choledocus. ou seja, a partir da confluência do ramus principalis dexter e do ramus principalis sinister. Não ocorre, portanto, tal como no relato das publicaçōes de JABL AN-PA.NTIC (1963). BASTOS NE'TO; PRADA'(1989). SOLZA ${ }^{1112}$ (1984. 1989). nos animais por eles estudados, a presença do ductus hepaticus. talvez implícito na observação de (OTTAVIANI" (1933), ao referir-se a este ducto de maneira indireta. admitindo linha divisória situada ao nível da contluência dos ducios hiliferos direito e esquerdo.

Por fim, nos cervos estudados, aparece em $25.0 \%$ dos casos isento de afluentes inominados.

\section{CONCIUSÕES}

Nas espécies Manzana americana. Blastocerus bezoarticus c Manzana simplicicornis integram o sistema do ramus principalis sinister, o ramus dorsalis lobi sinistri. o truncus intermediomedialis. o truncus intermediolateral. o ramus lateralis lobi sinistri o o ramus lobi quadrati.

Particularmente no Manzana americana. verificamos parlicipando do sistema do ramus principalis sinister. o ramus medialis lobi sinistri unido ao truncus intermediomedialis. em 50.0\% dos casos e o truncus intermediomedialis unido a) truncus intermediolateralis também em $50.0 \%$ das peças. () ramus lateralis lobi sinistri conllui para a via comum ao truncus intermediomedialis te o truncus intermediolaterais, em $33.3 \%$ das peças, constituindo tronco diretamente com este último, também em 33,3\% dos casos. c com o ramus lobi quadrati. $\mathrm{cm} 16.6 \%$ dos ligados.

O ramus medialis lobi sinistri unc-se ao ducto composto pelo truncus intermediomedialis. truncus intermediolateralis c ramus lateralis lobi sinistri $\mathrm{cm} 33.3 \%$ dos casos. enquanto o ramus lobi quadrati unce-se ao tronco formado pela confluência do ramus medialis lobi sinistri. truncus intermedionedialis mais truncus intermediolateralis e ramus lateralis lobi sinistri. em $50 \%$ das peças. () truncus intermediolateralis conflui para o tronco resultante da união do ramus medialis lobi sinistri c truncus intermediomedialis menos irequientemente.

No veado do rabo branco (Blastocerus bezoarticus). o ramus principalis sinister é formado a partir do ramus lobi quadrati que converge para tronco comum formado pelo ramus medialis lobi sinistri, truncus intermediomedialis. truncus intermediolateralis a ramus lateralis lobi sinistri. Este ducto acolhe alluente isolado do lobus caudatus pars supraportalis).

No veado catingueiro (Manzana simplicicornis), o ramus principalis sinister está composto pela união do truncus intermediodialis c truncus intermediolateralis. O ramus lateralis lobi sinistri, ramus dorsalis lobi sinistri alluem ao ducto formado pelos dois truncus c ramus lobi quadrati recebendo. uma ve\% assim constituído, afluentes do lobus caudatus (pars supraportalis) e lobus quadratus.

\section{SUMMARY}

The excretory system of the liver in 8 specimens of Manzana americana (6), 1 of Manzana simplicicornis (1) and 1 of Blastocerus bezoarticus (1) was injected with colored latex Neoprene. fixed in $10 \%$ formaldehyde solution and dissected. The ramus principalis sinister is made up of the ramus dorsalis lobi sinistri. the truncus intermediomedialis. the truncus intermediolateralis, the ramus lateralis lobi sinistri and the ramus lobi quadrati.

LNITERMS: Liver; deers: hiliary excretory system. 


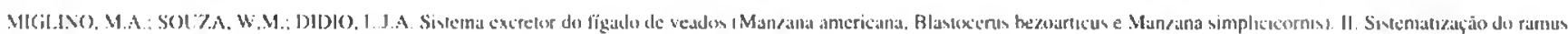

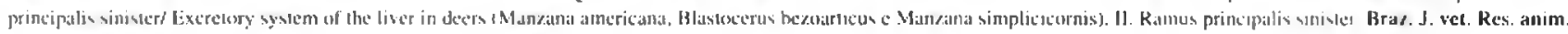
Sci.. Sio Pauls. V. 32. n. 4. p. 219.223.1945.

\section{REFERÊNCIAS BIBLIOGRÁFICAS}

I-ANNUNZIATA. M. Contribuição alo estudo das vias biliferas intra e extra hepáticas em ('apra hircus. Revista da Faculdade de Medicina Veterinária, São Paulo, v.8, p. $119-38,1969$.

2-BASTOS NETO. I.P.: PRAIDA, I.L.S. Contribuição â estudo das vias bilíferas de bovinos azebuados. Sistematização do ramus principalis sinister. Revista da Faculdade de Medicina Veterinária e Zootecnia da Universidade de São Paulo, v.20, p. 23-7, 1989.

3-I PERRICO, A.A. Contribuição ao estudo das vias biliferas intra e extra hepáticas em Capra hineus. Sistematização do ramus principalis dexter. Kevista da Faculdade de Medicina e Veterinária da Liniversidade de São Paulo, v. 13, p. 7.26. 1976.

4-GETTY, R. Sisson and Grossman's the Anatomy of the domestic animals. 5 ed. Philadelphia. WB Saunders. 1975, v.2, p. 913

5-JABLAN-PANTIC, C. Caracleristics and comparative ratios of intrahepatic bile duct in domestic animals. Acta Veterinária. Biograd. v. 13. p. 3-14. 1963.

6-OTTAVIANI, G. Ricerche radiographiche comparative sulle vie Bilifere Intrahepatiche. Atti del Istitut Veneto de Scienze, lellere ed arti,v. 92, p. 1065- 127, 1933.

7-PAIVA, O.M.: D'ERRICO, A.A. Aspectos analômi$\cos$ das vias bilíferas extra-hepáticas no carneiro. Folia Clinica et Biologica. São Paulo, v. 31, p. 37-40. 1959.

8-PRADA. I.L.S. Contribuição ao estudo das vias bilíteras em ovinos (Ovis aries Linnaeus, 1758). Sistematìção do ramus principalis sinister. Vias bilíferas da girafa (Giraffa camelopardalis). Revista da Faculdade de Medicina Veterinária e Zootecnia da Universidade de São Paulo, v. 12, p. 45-52. 1971.

9-PRADA, I.L.S.: BORELLI. V.: PEDUTI NI:TO. J. Contribuição ao estudo do sistema excretor do fígado em animais silvestres. 1 - Vias bilíferas da girafa (Cirafta camelopardalis). Revista da Faculdade de Medicina Veterinária e Zootecnia da Ĺniversidade de São Paulo.v. 12. p. $45-52.1976$.

10-PRADA. I.L.S.; IERRICO. A.A.; FE.RNANIDI:S FIL.HO, A.; PEREIRA. J.G.L. Contribuição ao estudo das vias bilíleras em ovinos deslanados. In: CONGRliSSO BRASILEIRO DE: ANATOMIA. 14, Vitória. Anais. p. 79. 1985.

I ]-SOUZA, W.M. Contribuição ao estudo das vias biliferas intra e extra-hepáticas em bovinos da raça Nelore. São Paulo, 1984. Tese (Doutorado) - Instituto de Ciências Biomédicas, Universidade de São Paulo.

12-SOUZA. W.M. Estudo anatômico sobre as vias bilíferas intra e extra hepáticas em búfalos. Jaboticabal. 1989. Tese (Doutorado) Faculdade de Ciências Agrárias e Veterinárias, Campus de Universidade Estadual Paulista.

1.3-SOLZA, W.M.; MIGLINO, M.A.; PRADA. I.L.S.; D'ERRICO. A.A. Contribuição ao estudo das vias bilíferas intra e extra-hepáticas em ovinos (Ovis aries Limnaeus 1758) da raça Ideal. In: CONGRESSO BRASILEIRO DE ANATOMIA, 14, Vitória. Anais. p. 78. 1985.

Recebido para publicação em 8/9/93 Aprovado para publicação em 13/2/95 